rarely (never, to our knowledge) attempt to correct efficacy estimates for undetected or subclinical infections through the use of post-trial serology. They also, to our knowledge, rarely use serostatus at the time of enrollment to stratify analyses, despite recommendations for such analyses in clinical trial guidelines ${ }^{12}$. For COVID-19, there is little basis on which to predict vaccine efficacy, as no vaccine against coronavirus has been tested for efficacy.

Vaccines are also typically tested for efficacy either in populations with low baseline immunity (e.g., vaccines against measles, for infants) and/or for diseases for which natural immunity is partial and/or short-lived, so it would be difficult to measure (e.g., pneumococcal vaccines) ${ }^{13}$. Nonetheless, simulations have shown that such trials can be complicated by the interplay between naturally induced immunity and vaccine-induced immunity ${ }^{14}$. For the Dengvaxia vaccine against dengue fever, researchers gathered but did not immediately analyze serological samples from trial participants. After publication of the original trial results, a secondary analysis of the data showed that the vaccine was most beneficial for people with a prior seropositivity to infection with dengue virus, but that if a person received Dengvaxia while seronegative to infection with dengue virus, the vaccine could prime an 'enhancement' of infection that made the symptoms more severe ${ }^{15}$. This critical aspect of vaccine safety and efficacy could not have been fully understood if serum samples had not been available.

Serological measurement of infection at the end of a vaccine trial is especially important for infections for which asymptomatic infections are very important. This includes infections such as infection with SARS-CoV-2, in which asymptomatic transmission contributes to spread, or infections such as infection with Zika virus, in which asymptomatic infection can produce sequelae with potentially severe consequences, such as congenital Zika syndrome. Serological measurement also matters when failing to do so biases efficacy measurement downward. In sum, serology at the start and end of trials should be more common than it is.

\section{Conclusion}

Clinical trials are being set up at a rapid rate to test various approaches to preventing COVID-19. Getting fully interpretable and unbiased results from these trials depends on serological testing of participants at baseline and (for at least a subset of the participants) ${ }^{7}$ at the end of the trial. While accurate serological tests are still in development, trialists have a window of opportunity for obtaining blood from trial participants and banking it in anticipation of having such tests in the near future. It is essential that this opportunity be taken in order to maximize the scientific value of the information that these trials provide.
Marc Lipsitch $\left(\mathbb{D}^{1,2}{ }^{凶}\right.$, Rebecca Kahn (D) ${ }^{1}$ and Michael J. Mina ${ }^{1,2}$

${ }^{1}$ Center for Communicable Disease Dynamics, Department of Epidemiology, Harvard T.H. Chan School of Public Health, Boston, MA, USA. ${ }^{2}$ Department of Immunology and Infectious Diseases, Harvard T.H. Chan School of Public Health, Boston, Mam, USA.

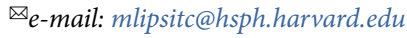

Published online: 27 April 2020

https://doi.org/10.1038/s41591-020-0887-3

References

1. Feng, S. et al. Lancet Respir. Med. https://doi.org/10.1016/ S2213-2600(20)30134-X (2020).

2. Lurie, N., Saville, M., Hatchett, R. \& Halton, J. N. Engl. J. Med. https://doi.org/10.1056/NEJMp2005630 (2020).

3. Kimball, A. MMWR Morb. Mortal. Wkly. Rep. 69, 377-381 (2020)

4. Bao, L. et al. bioRxiv https://doi.org/10.1101/2020.03.13.990226 (2020).

5. Callow, K. A., Parry, H. F., Sergeant, M. \& Tyrrell, D. A Epidemiol. Infect. 105, 435-446 (1990).

6. Hernández, A. V., Eijkemans, M. J. C. \& Steyerberg, E. W. Ann. Epidemiol. 16, 41-48 (2006).

7. Kahn, R., Hitchings, M., Wang, R., Bellan, S. E. \& Lipsitch, M. Am. J. Epidemiol. 188, 467-474 (2019).

8. Welliver, R. et al. J. Am. Med. Assoc. 285, 748-754 (2001)

9. Sivay, M. V. et al. J. Acquir. Immune Defic. Syndr. 75, 271-279 (2017).

10. Sukwa, T. Y., Mulenga, M., Chisdaka, N., Roskell, N. S. \& Scott, T. R. Am. J. Trop. Med. Hyg. 60, 521-525 (1999). 11. Cowling, B. J. et al. Ann. Intern. Med. 151, 437-446 (2009). 12. World Health Organization. https://www.who.int/biologicals/ expert_committee/Clinical_changes_IK_final.pdf (2016). 13. Black, S. et al. Pediatr. Infect. Dis. J. 19, 187-195 (2000).

14. Cai, F. Y., Fussell, T., Cobey, S. E. \& Lipsitch, M. PLoS Comput. Biol. 14, e1006333 (2018).

15. Sridhar, S. et al. N. Engl. J. Med 379, 327-340 (2018)

\title{
An overdose surge will compound the COVID-19 pandemic if urgent action is not taken
}

\author{
In the USA and around the world, the COVID-19 pandemic arrived as the population was fighting a devastating \\ opioid overdose epidemic. Urgent and decisive action is needed to protect particularly vulnerable populations, \\ such as those with opioid use disorder, to prevent a compounding effect on public health.
}

\section{Sarah E. Wakeman, Traci C. Green and Josiah Rich}

\footnotetext{
$\Lambda$ s the global spread of the coronavirus SARS-CoV-2 continues, the pandemic's impact on people who are already marginalized and vulnerable will be profound, and the 'knock-on' effect on public health will be severe. This is particularly true of people with opioid use disorder.

wCounterintuitively, the fact that social-distancing measures are leading to perturbations in the illegal drug market,
}

making it harder to access, could have negative effects on public health down the line. Richard Cowan's 'iron law of prohibition' dictates that under stricter regulation, illegal providers will produce more-powerful product because it takes up less space and is more easily transportable. Recently, this has driven the shift from heroin to fentanyl in the USA's illegal market ${ }^{3}$. Moreover, it is important to note that opioid use leads to tolerance of opioids. After weeks of reduced access to opioids, tolerance decreases, and once those with opioid use disorder gain access to opioids again, they are more likely to overdose. To compensate for lack of access to opioids, people with opioid use disorder may also seek other substances, such as alcohol and benzodiazepines, both of which potentiate overdose risk. These supply-side pressures, which result in an unpredictable 
and riskier product, combined with reduced tolerance, make each episode of use more likely to result in overdose ${ }^{4}$.

This challenging predicament, however, could offer opportunity. Poor access to illegal opioids may drive those who were undecided about use of methadone or buprenorphine to seek treatment. These medications are the most effective treatment we have for opioid use disorder. They successfully reduce craving, withdrawal, drug use, overdose and acute-care utilization ${ }^{5}$.

Despite their effectiveness, these medications are underutilized, and most treatment facilities and healthcare settings do not make them easily available. To not immediately pursue a scale-up of availability and outreach to encourage people with opioid use disorder to engage with buprenorphine and methadone treatment would be foolhardy.

Due to the COVID-19 emergency, the US federal government has temporarily waived the initial in-person assessment for initiation of buprenorphine and has increased flexibility for the dispensation of take-home methadone. These changes allow prescribers to initiate buprenorphine treatment remotely. These changes are welcome, and the federal government should do more along these lines. All providers with prescriptive authority should be allowed to prescribe buprenorphine, which could be achieved by removal of the requirement for a US Drug Enforcement Administration ' $\mathrm{X}$ ' waiver. Emergency funding for buprenorphine and methadone should be released so that patients who are unable to afford these treatments, particularly in states without Medicaid expansion, can access treatment. Importantly, the structure of treatment settings themselves could increase infectious spread without thoughtful redesign. Limiting requirements for frequent in-person visits, facilitating remote healthcare delivery and providing these healthcare providers with appropriate protective equipment would be paramount to preventing the further spread of

\section{SARS-CoV-2.}

Pharmacies should also be better used to provide a more normalized pathway to care and align with social-distancing goals. They should be allowed to dispense methadone, and reimbursable prescriber-pharmacist collaborations that allow prescribers to delegate buprenorphine prescribing to pharmacists should be encouraged. Postacute-care facilities such as skilled-nursing facilities should also be made exempt from regulations related to methadone and buprenorphine so that, like an acute-care hospital, these facilities could administer medications for opioid use disorder. This last change is particularly important as hospitals face capacity surges related to COVID-19 and need to move patients to lower acuity settings. At the same time that we pursue these new avenues to increase access to methadone and buprenorphine, we must continue broad access to proven harm-reduction interventions such as naloxone distribution and syringe-service programs.

Crisis leads to opportunity. COVID-19 presents unique and urgent challenges. However, it also presents an opportunity to create a healthcare system that truly addresses the needs of vulnerable populations, such as those with opioid use disorder. We must first act quickly to stave off a substantial increase in overdoses, an impending crisis on top of a crisis.

\section{Sarah E. Wakeman ${ }^{1,2}$, Traci C. Green ${ }^{3,4}$ and Josiah Rich ${ }^{4,5}$ \\ ${ }^{1}$ Department of Medicine, Massachusetts General Hospital, Boston, MA, USA. ${ }^{2}$ Harvard Medical School, Boston, MA, USA. ${ }^{3}$ The Heller School for Social Policy and Management,Brandeis University, Waltham, MA, USA. ${ }^{4}$ The Warren Alpert School of Medicine of Brown University, Providence, RI, USA. ${ }^{5}$ The Center for Prisoner Health and Human Rights at The Miriam Hospital, Providence, RI, USA.

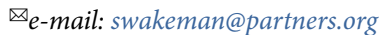

Published online: 1 May 2020

https://doi.org/10.1038/s41591-020-0898-0

References

1. John, W. S. et al. Drug Alcohol Depend. 194, 468-475 (2019).

2. Akiyama, M. J., Spaulding, A. C. \& Rich, J. D. N. Engl. J. Med. https://doi.org/10.1056/NEJMp2005687 (2020).

3. Beletsky, L. \& Davis, C. S. Int. J. Drug Policy 46, 156-159 (2017).

4. Ciccarone, D. Int. J. Drug Policy 71, 183-188 (2019).

5. Wakeman, S. E. et al. JAMA Netw. Open 3, e1920622 (2020).

\section{Acknowledgements}

The input of T.C.G. and J.R. to this manuscript was funded, in part, by US National Institutes of Health grant number P20GM125507. S.E.W. has received research support through Massachusetts General Hospital from the National Institutes of Health (1R01DA044526-01A1), the National Institute on Drug Abuse (3UG1DA01583117S2), the Substance Abuse and Mental Health Services Administration (1H79TI081442-01) and the Laura and John Arnold Foundation, and received salary support from OptumLabs as a co-investigator on a research project.

\section{EHRs could clarify drug safety in pregnant people}

Testing drug safety in people who are pregnant remains a wicked problem, but in the transition toward big data and machine learning, target trials could afford a viable alternative to randomized, controlled trials.

\section{Anup P. Challa, Robert R. Lavieri, Ethan S. Lippmann, Jeffery A. Goldstein, Lisa Bastarache, Jill M. Pulley and David M. Aronoff}

$\mathrm{n}$ the USA, concern over the 'thalidomide disaster' of the late 1950s and early 1960s led the US Food and Drug Administration to increase its oversight of drug exposures during pregnancy and to develop a five-point scale to rank the teratogenicity of a drug, which had the consequence of prohibiting pregnant people from participating in drug safety trials for ethical reasons. Consequently, there has been a lack of data on drug safety for pregnant people, and prescriptive behavior for those who are pregnant is guided by often conflicting or poorly informative results from pre-clinical studies. Paradoxically, this has increased both the risk for adverse drug outcomes and the under-treatment of those who are pregnant, and several diseases of pregnancy remain 'orphaned'1. This situation is similar elsewhere in the world; although in this Comment, we focus on the effects of regulatory policies of the USA, the European Medicines Agency has implemented similarly exclusionary policies for the participation of pregnant people in clinical trials in the European Union ${ }^{2}$. This seems unacceptable, particularly in light of 